\title{
Author Index Vol. 208,1994
}

Adachi-Usami, E. 220

Aikawa,Y. 284

Alatsaki, M. 71

Aliseda-Pérez de Madrid, D. 115

Altamirano, D. 49

Altan,T. 318

Arocker-Mettinger, E. 314

Arvanitis, P. 71

Ayaki,M. 110

Azad,R.V. 207

Baldi,A. 37

Battaglia Parodi, M. 29,172 Baudouin,C. 309 Baudrimont, M. 289 Bavbek,T. 318 BehrensBaumann, W. 32 Berio,A. 342 Bertelli,E. 321 Biavasco, F. 321 Bonavolontà, G. 329 Boscaino, A. 329 Brancato, R. 239 Bredehorn,T. 98 Bucher, P.J.M. 10

Çag $1 / 8$ r, Y. 112 Calderini,S. 172,179 Capoferri,C. 15 Cappelli,S. 304 Caramazza, N. 37

Carpineto,P. 247 Cascone, G. 192 Casellato, L. 95 Casi,P. 304 Castagna, I. 230 Ceccuzzi, R. 243

Cellini, M. 37 Cennamo,G. 198,329 Chemelli,A. 195 Chin,S. 168 Ciancaglini, M. 247

Claeys,M. 49 Corcóstegui, B. 163

Damanakis, A. 71 Daxer,A. 195 De Felice, G.P. 37 Degn,T. 147 DelPrete,A. 295,333

DeNatale,R. 10 Denis, P. 289 DeRosa, G. 329

Di Giovanni, A. 295 Duncker, G.I.W. 98, 259, 262

Egger, S.F. 77 Elenius, V. 136 Elibol,O. 112 Ersöz,C. 270 Ertl,A. 119 Ettl,A. 195

Famà, F. 230 Felber,S. 195 Ferrante, M. 333 Ferri,R. 192 Filippello, M. 192 Fischer-Klein, C. 195 Flammer,J. 10,142,336 Frongia,G.B. 321 Fujii,M. 339 Fujiwara,N. 131,254 Fukuo,Y. 54,267

Galassi,F. 304 Gallenga, P.E. 247 Garcia-Arumí, J. 163 Gaspar,A.Z. 142 Gass, A. 336

Gastaud,P. 309 Gazzaniga, A. 37 Gelmi,C. 243 Giovannini, A. 185,321 Gómez-Demmel, E. 115

Grabner,G. 77 Grille, W. 259 Gündüz, K. 61

Haciyakupoglu, G. 270

Haciyakupoglu, S. 270

Haruno,I. 298

Hashimoto, M. 226

Hatono, N. 298

Hayami, H. 54

Hayasaka, S. 46, 145, 175, 210, 284, 339

Heegaard, S. 82

Hendrickson, Ph. 10,142

Herbort,C.P. 49

Hirata,H. 54,267 
Holbach,L. 65

Honda, Y. 250

Hoya,T. 122

Huber-Spitzy, V. 77,314

Igarashi,Y. 201,226 Ikeda,H. 220 Ikonomopoulos, N. 71 Inomata, H. 157 Ishibashi,T. 157

Ishigouoka, H. 250 Ishikawa, S. 298 Isola, V. 29,172

Kadoi,C. 267 Kaiser, HJ. 10 Katayama, T. 54,267 Kato,T. 267 Kawamoto, K. 210 Kawano,S.-I. 250 Kaya,M. 270 Kazokoglu,H. 318 Kimmich, F. 32 Kimura,T. 220 Kinoshita, M. 250

Kisters, G. 259 Kitz,K. 119 Kodama,T. 210,284 Kollegger, E. 92 Krausse, R. 262 Kubo,C. 131

Kubota,Y. 267 Küchle,M. 65 Kumar, A. 207 Kuper, H. 98 Kurihashi,K. 216 Kurome,H. 175

Kusunoki,S. 105

Lajara-Blesa, J. 115 Langmann, A. 92 Laroche, L. 289 Lee,S.-M. 17 Leinonen, M.T. 136 Liang, R.-C. 17 Lin,S.-Y. 17 Lindner, S. 92 Lobefalo,L. 247 Loffredo,C 333 Lue, J. 32

Maekawa, H. 201 Manso,E. 321 Mäntyjärvi, M. 23 Mariotti,C. 185,321 Martorina, M. 15

Masciello, M. 295, 333 Mastropasqua, L. 247

Mateo,C. 163 Matsuda,A. 168 Menapace, R. 119 Menga, M. 15 Mihara, S. 46 Minagawa, H. 157 Minamoto, A. 105 Minicucci, M. 239 Mito,Y. 226 Miyagawa, M. 46, 142 Miyazaki, M. 122 Moreno-Montañés, J. 115 Mori,R. 157

Nagaoka, S. 46 Nakamura, Y. 226 Nameki,H. 110 Naumann, G.O.H. 65 Negi,A. 250

Nishimoto,H. 298 Nishimura, M. 233 Noda,S. 145,175,210,284 Nordmann, J.-P. 289 Nuzzaci, G. 304

Ogasawara, K. 201 Oguchi,Y. 110 Ohashi,T. 168 Ohba,M. 201 Ohde,H. 110 Ohnishi,Y. 233

Ohwada,Y. 226 Ohyachi,H. 201 Orgül,S. 142,336 Oyachi,H. 226 Özdemir,N. 270 Özdemir, Ö. 61

Palmowski, A. 44 Palomba, M. 179 Papst,N. 98 Pece,A. 239 Piazzi,A. 342

Pierro, L. 239

Polat, S. 270

Ravalico,G. 179 Reccia, R. 295 Reich, U. 262 Ricci,R. 230 Rieger, G. 5 Ripa,E. 185 Rochat,C. 49 Rosa,N. 198,329 Rosenwasser, G.O.D. 198 Rouras, A. 163 Ruprecht, K.W. 44

Saeki, M. 220 Sagara,T. 254 Sakai,Y. 157 Sakata,H. 105,325 Salmeri,G 230 Salvador, F. 163 Saraux,H. 289 Scala, A. 295

Scassellati Sforzolini, B. 185 Schneider, B. 77 Scholda,C. 77 Scimone, G. 192 Sebastiani, A. 198,295,333 Sepe,G 333 Seth,M. 207 Sharma,Y.R. 207 Shigeto,M. 233 Shirakawa,S. 298

Sirianni, P. 15 Sodi,A. 304 Steinkogler, FJ. 314 Stümpfig,D. 10 Suzuki, R. 131,254 SuzukiYoshida, S. 250

Takeda,N. 54,220,267 Tamura, T. 41 Tanaka, H. 233

Tanaka,K. 131,254 Tanihara, H. 250 Temel,A. 318 Tewari,H.K. 207 Tezuka,T. 41,161

Tiemann, M. 65 Tittarelli, R. 185,321 Tofukuji, S. 1 Tognetto,D. 179 Tracló,C.D. 230 Tranfa,F. 329 Trombetta, C.J. 230 Tsubota,K. 214 Tsumura,K. 105,325 Tuncer, í. 270 Tuppurainen, K. 23 Uchida,H. 233 Ueda,Y. 250 Uga,S. 298 Urbak,S.F. 147 Uto,H. 233

Vattovani, O. 179 Vécsei,V.P. 314 Vielmo,A. 304

Wada,M. 284 Walt,J.G. 32 Watanabe, M. 325 Welsh, R.C. 344 Winkler,R. 5

Yamada,M. 214 Yamamoto, M. 105 Yoneyama, J. 278 Yoshida, M. 41,161 Yüksel,N. 112

Zagami, A. 192 Zehetmayer, M. 119 
346

Author Index 